\title{
SIR Model based Study on Chicken Pox Outbreak in Kamrup(M) District of Assam, India
}

\author{
Rima Devi \\ Department of Information Technology \\ Gauhati University \\ Assam-781014, India
}

\author{
Balendra Kumar Dev Choudhury \\ Pub Kamrup College, Baihata Chariali, Kamrup \\ Assam-781381, India
}

\begin{abstract}
In this paper, a mathematical model based on SIR (susceptible-Infected-Removed) model has been developed to study various parameters related to infectious diseases in Kharghuli and Hengrabari area of Guwahati city of Assam, India where an outbreaks of chicken pox occur during April 2017. From the modeling, the basic reproductive ratio for chicken pox is found to be less than unity. This modeling will help to predict the disease spreading as well as to take preventing measure of disease treatment in various locality of Assam, India.
\end{abstract}

\section{General Terms}

Mathematical model, Chicken Pox, Disease spreading

\section{Keywords}

Mathematical model, Infectious disease, Parameters Ratio

\section{INTRODUCTION}

Mathematical biology is a highly interdisciplinary area. It is the application of mathematical modeling to solve problems in biology and physiology. Mathematical epidemiology is one of the oldest and richest areas in mathematical biology. The study of causes of occurrence and transmission of diseases in human population is considered as epidemiology. The use of mathematical model in the study of infectious diseases helps in estimation of parameters of biological significance and deductions of concepts not directly accessible from the data.

Daniel Bernoulli is regarded as the father of epidemiological modeling as he investigated the influence of variation on life expectancy as early as 1760 .One of the fundamental principles of epidemiology was established by Kermack and McKendrick in 1927.

Encouraging by the various studies on needs of mathematical model to prevent disease spreading, in this work, mathematical model is developed to predict various parameters of chicken pox spreading in the Kharghuli area of Kamrup(M) district of Assam in the country India. Using the SIR model, basic reproductive ratio, Herd Immunity Threshold, control vaccination number and effective reproductive number is analyzed.

The paper is organized as follows: Section 1 is an introduction. The SIR model formulation is discussed in the next section. In section 3, A case-study based scenario for the spread of chickenpox is described (based on interviews and direct observations) and found out the basic reproductive ratio and herd immunity threshold .Finally, A concluding discussion considers what assistance might be necessary if rural communities are to reduce transmission rates.

\section{METHODS}

\section{1 sir Model}

Among all the epidemiological model, SIR model [Getz, Wayne M., and James O. Lloyd-Smith (2005)] is one of the simplest and fundamental model. Considering that the population consists of three types of individuals, denoted by the letters S, I and R (which is why this is called an SIR model) and all are functions of time $t$ which can be changed according to a system of differential equations, following assumptions are made to develop the present SIR model:

1) The population is fixed in the area.

2) A person can leave the susceptible group if he or she is infected. A person can leave the infected group if he or she is recovered from the disease. If the person has recovered once from the disease, the person received immunity.

3) The probability of being infected from the disease does not depend on factors like age, sex, social status, race.

4) There is no inherited immunity of the people.

5) The people are mix homogeneously.

The model starts with the following notations:

$\mathrm{S}(\mathrm{t})$, the number of susceptible individuals at time $\mathrm{t}$

$\mathrm{I}(\mathrm{t})$, the number of infected individuals at time $\mathrm{t}$

$\mathrm{R}(\mathrm{t})$, the number of recovered individuals at time $\mathrm{t}$

$\& \mathrm{~N}$ the total population size.

The assumptions can lead us to a set of differential equations:

$\mathrm{dS} / \mathrm{dt}=-\beta \mathrm{S}(\mathrm{t}) \mathrm{I}(\mathrm{t})$

$\mathrm{d} I / \mathrm{dt}=(\beta S(\mathrm{t})-\mathrm{K}) \mathrm{I}(\mathrm{t})$

$\mathrm{dR} / \mathrm{dt}=\mathrm{KI}(\mathrm{t})$

Here $\mathrm{k}$, the rate of recovery (with greater or equal to zero), $\propto$ ,the probability of becoming infected, $\gamma$, the number of people infected person comes in contact within each period of time on average, $\beta$, the average number of transmissions from an infected person in a time period(with $\beta$ greater or equal to zero), and

$\mathrm{S}(\mathrm{t})+\mathrm{I}(\mathrm{t})+\mathrm{R}(\mathrm{t})=\mathrm{N}$

By applying Euler's method of systems, we can solve the

Differential equations. The solutions to the differential equations are:

$\mathrm{Sn}+1=\mathrm{Sn}-\beta \mathrm{SnIn}$ 
$\operatorname{In}+1=\operatorname{In}(1+\beta \operatorname{Sn}-\mathrm{K}) \Delta \mathrm{t}$

$\mathrm{Rn}+1=\mathrm{Rn}+\mathrm{KIn} \Delta \mathrm{t}$

where $\mathrm{Sn}+1, \mathrm{In}+1$ and $\mathrm{Rn}+1$ are the number of susceptible, infected and recovered people at time $(n+1)$.

\subsection{Basic Reproductive Ratio}

An important part of disease modeling is $B_{R}$, called as Basic Reproductive Ratio[Bauch,Chris, and David Earn(2003)]. The Basic Reproductive Ratio is an important parameter since it tells us if a population is at risk from a disease. $B_{R}$ is affected by the infection and removal rates, i.e. $\beta,, \mathrm{K}$, and is obtained by $B_{R}=\beta K$. When $B_{R}>1$, the occurrence of the disease will increase. When $B_{R}<1$, the occurrence of the disease will decrease and the disease will eventually be eliminated. When $\mathrm{B}_{\mathrm{R}}=1$, the disease occurrence will be constant [Rahman, S M Ashrafur (2016)].

\subsection{Herd Immunity Threshold}

The Herd Immunity Threshold is percentage of the population that needs to be immune to control transmission of a disease, i.e. equal to one. The equation given by Diekmann and Heesterbeekfor figuring out the Herd Immunity Threshold is

$\mathrm{Ht}=\mathrm{B}_{\mathrm{R}}-1 / \mathrm{B}_{\mathrm{R}}$

As the amount of vaccinations increase, the herd immunity threshold also increases. By decreasing the amount of susceptible people, the herd immunity threshold decreases.

MATLAB is used as the computational tool for the present investigations

\section{RESULTS AND DISCUSSION}

Chickenpox is a highly contagious disease. The risk of complications depends on age and immune status. Source of infection is a person with manifest or in apparent infection. The disease is transmitted from person to person by direct contact and droplets. Incubation period ranks from 14 to 21 days. The virus is highly contagious and can be spread 1-2 days before the rash appears until all blisters form crusts (usually 3-7 days).

Here, an experimental survey results for the population up to 1400 people that mix homogeneously for Kharghuli area of Kamrup(M) district of Assam, India has been conducted during the outbreak of chicken pox disease(during MayJune,2017) and the modeling for chicken pox has been done using SIR model.

In tables 1 and 2 cases are calculated, where one can see how many people will be in each state at a given period of time for Kharghuli and Hengrabari area respectively.

Table 1: State of people vs. time period for Kharghuli area

\begin{tabular}{|c|c|c|c|}
\hline \multirow{2}{*}{$\begin{array}{c}\text { Time } \\
\text { Period } \\
\text { (in days) }\end{array}$} & \multicolumn{3}{|c|}{ State } \\
\cline { 2 - 4 } & $\mathrm{S}$ & $\mathrm{I}$ & $\mathrm{R}$ \\
\hline 0 & 1275 & 0 & 0 \\
\hline 1 & 1274 & 1 & 0 \\
\hline
\end{tabular}

\begin{tabular}{|c|c|c|c|}
\hline 2 & 1257 & 3 & 0 \\
\hline 3 & 1202 & 3 & 1 \\
\hline 4 & 1100 & 1 & 0 \\
\hline 5 & 1000 & 0 & 0 \\
\hline 6 & 900 & 0 & 0 \\
\hline 7 & 890 & 1 & 2 \\
\hline 8 & 400 & 1 & 5 \\
\hline 9 & 100 & 0 & 2 \\
\hline
\end{tabular}

From Table 1 and 2,one can calculate $\beta$.To do this one manipulate equation (5) using $\Delta \mathrm{t}=1$ to get the following

$$
\beta=\frac{S n-S n+1}{S n I n}
$$

Using this equation one can get the value of for each period. These are shown in the tables $3 \& 4$.

Table 3: The different $\beta$ 's for each period and the average $\beta$ for Kharghuli area

\begin{tabular}{|c|c|}
\hline Period $(n+1)$ (in days) & $\beta$ \\
\hline 0 & 0 \\
\hline 1 & 0.0133437991 \\
\hline 2 & 0.1312649165 \\
\hline 3 & 0.2545757072 \\
\hline 4 & 0.0909090909 \\
\hline 5 & 0 \\
\hline 6 & 0 \\
\hline 7 & 0 \\
\hline 8 & 0.5505617978 \\
\hline 9 & 0 \\
\hline Average $\beta$ & 0.1040655313 \\
\hline
\end{tabular}


Table 2: State of people vs. time period for Hengrabari area

\begin{tabular}{|c|c|c|c|}
\hline \multirow{2}{*}{$\begin{array}{c}\text { Time } \\
\text { Period (in days) }\end{array}$} & \multicolumn{3}{|c|}{ State } \\
\hline & $S$ & I & $\mathrm{R}$ \\
\hline 0 & 1590 & 1 & 0 \\
\hline 1 & 1580 & 0 & 0 \\
\hline 2 & 1550 & 0 & 0 \\
\hline 3 & 1500 & 1 & 1 \\
\hline 4 & 1450 & 0 & 0 \\
\hline 5 & 1400 & 1 & 0 \\
\hline 6 & 1350 & 3 & 0 \\
\hline 7 & 1300 & 0 & 0 \\
\hline 8 & 1250 & 0 & 0 \\
\hline 9 & 1200 & 0 & 4 \\
\hline 10 & 1150 & 0 & 0 \\
\hline 11 & 1100 & 0 & 0 \\
\hline 12 & 1050 & 0 & 0 \\
\hline 13 & 1000 & 2 & 0 \\
\hline 14 & 950 & 1 & 2 \\
\hline 15 & 900 & 1 & 1 \\
\hline 16 & 850 & 2 & 2 \\
\hline 17 & 800 & 2 & 2 \\
\hline
\end{tabular}

Table 4: The different $\beta$ 's for each period and the average $\beta$ for Hengrabari area

\begin{tabular}{|c|c|}
\hline Period(n+1) (in days) & $\beta$ \\
\hline 0 & 0 \\
\hline 1 & 0 \\
\hline 2 & 0.03333 \\
\hline 3 & 0 \\
\hline 4 & 0.035714 \\
\hline 5 & 0.1111 \\
\hline 6 & 0 \\
\hline 7 & 0 \\
\hline 8 & 0 \\
\hline 9 & 0 \\
\hline 10 & 0 \\
\hline 11 & 0 \\
\hline 12 & 0.025 \\
\hline 13 & 0.052631 \\
\hline 14 & 0.055555 \\
\hline 15 & \\
\hline
\end{tabular}

\begin{tabular}{|c|c|}
\hline 16 & 0.1176470588 \\
\hline 17 & 0.03125 \\
\hline Average $\boldsymbol{\beta}$ & $\mathbf{0 . 0 2 5 6 7 9 2 8 1}$ \\
\hline
\end{tabular}

Fig. 1 shows the rate of suspected, infected and recovered people in that particular time period. Similarly, for Hengrabari area of Kamrup(M) district of Assam, India the modeling for chicken pox spreading has been done based on survey results and is shown by Fig.2.

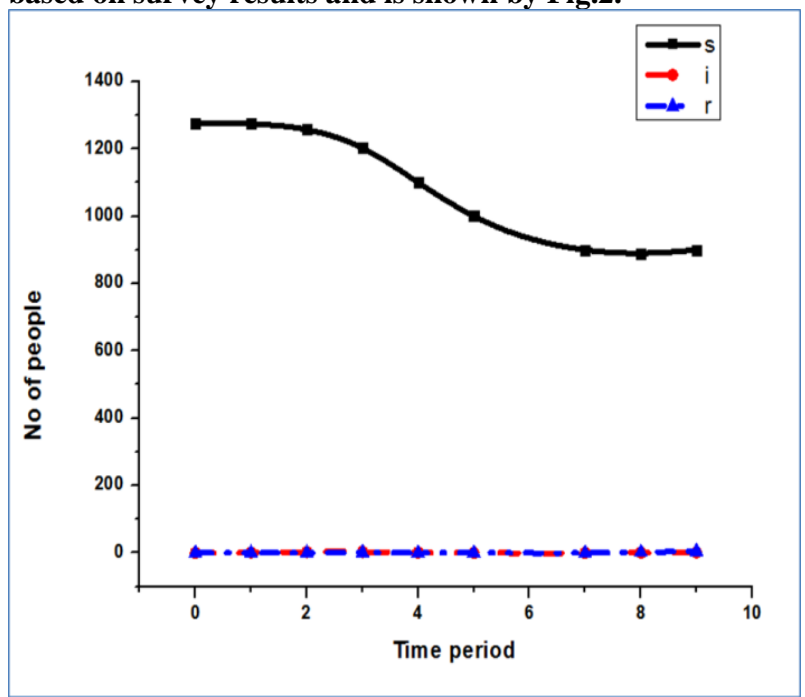

Fig.1: The effect of chicken pox during the period of outbreak in Kharghuli area of Kamrup(M),Assam,India.

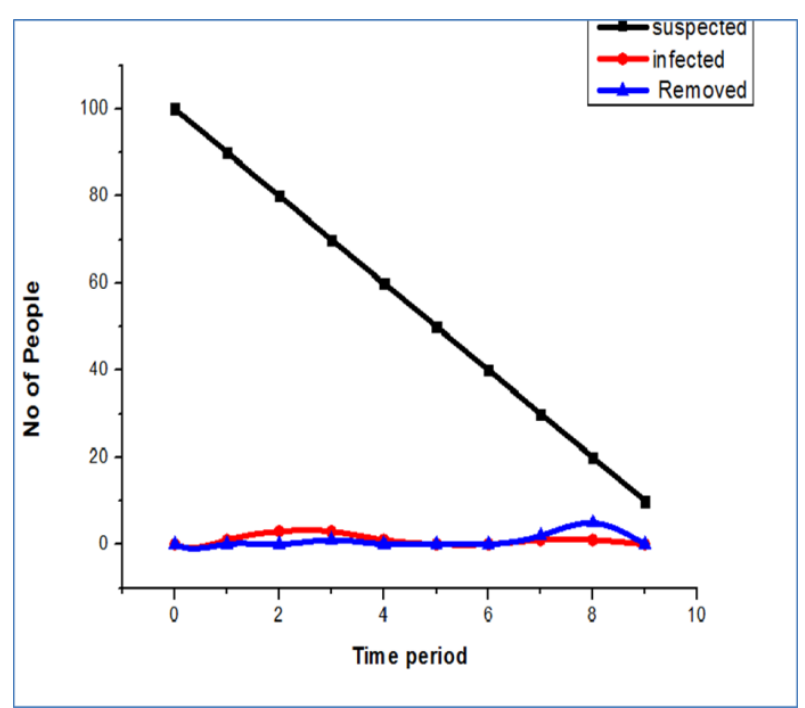

Fig.2: The effect of chicken pox during the period of outbreak in Hengrabari area of Kamrup (M), Assam, India.

In both the cases, the infected and recovered rate is same i.e. the number of people infected are all recovered.

From figure1, it is seen that the infected and recovered number is constant for Kharghuli area during the whole outbreak time period but for the Hengrabari area, the recovered rate in 7 to 9 days are more where as in 2 to 3 days, the rate of infection is more compared to rate of recovery which is seen in figure 2 . Thus proper modelingof real time data can give a concrete structure of the diseases spreading. The rate of transmission during the period of outbreak is also calculated and is shown in Fig. 3 and Fig.4 for Kharghuli area and Hengrabari area, respectively. For each period $\beta$ is 
different and there is a particular time band in which for both the cases, $\beta$ is maximum.

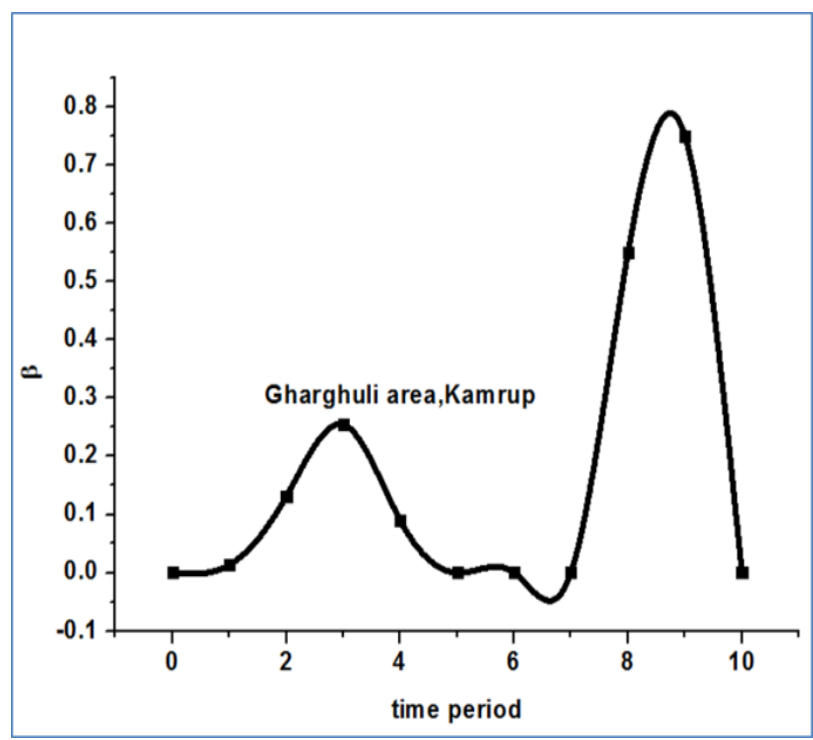

Fig. 3: The rate of transmission vs. time period for Kharghuli area, Kamrup,Assam,India.

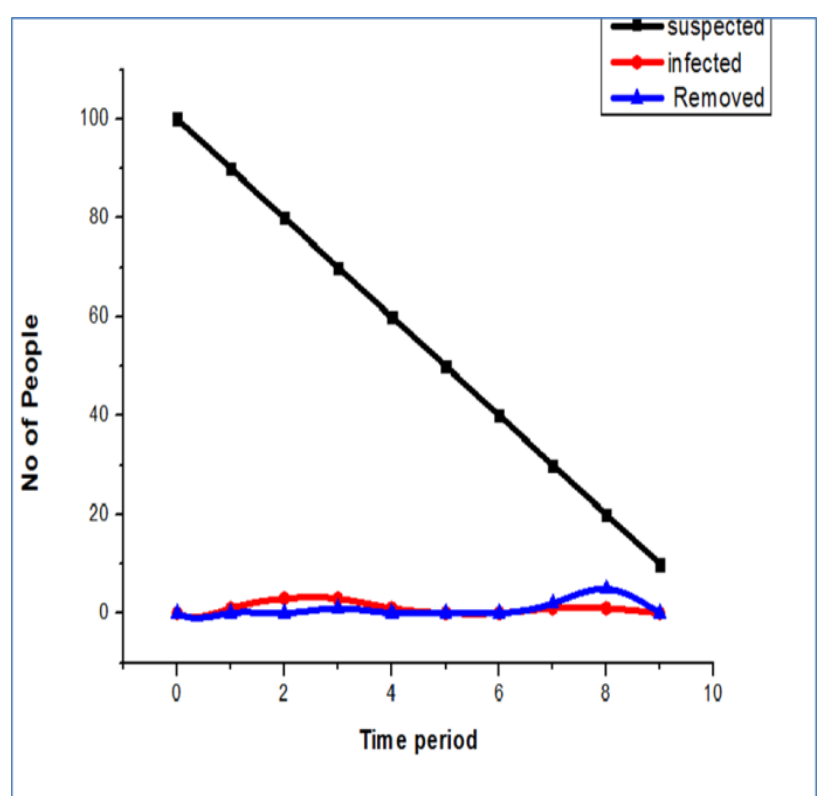

Fig. 4: The rate of transmission vs. time period for Hengrabari area, Kamrup, Assam, India.

\subsection{Basic Reproductive Ratio For \\ Chickenpox}

For the outbreak of chickenpox, we calculated the Basic Reproductive Ratio as

$\mathrm{B}_{\mathrm{R}}=\beta / K$

$=0.1040655313 / 1$

$=0.1040655313$ (For Kharghuli area)

For Hengrabari area,

$$
\begin{aligned}
& \mathrm{B}_{\mathrm{R}}=\beta / K \\
&=0.025679281 / 1
\end{aligned}
$$

$=0.025679281$

\subsection{Herd Immunity Threshold of chicken pox}

Since the Basic Reproductive Ratio is calculated, we can now calculate the Herd Immunity Threshold.

For Kharghuli area, herd immunity threshold is given below-

$\mathrm{H}_{1}=\frac{\mathrm{BR}-1}{\mathrm{BR}}=\frac{0.1040655313-1}{0.1040655313}=-8.609329696$

For Hengrabari area, herd immunity threshold is given below

$\mathrm{H}_{1}=\frac{\mathrm{BR}-1}{\mathrm{BR}}=\frac{0.025679281-1}{0.025679281}=-37.94190028$

From the present study, the obtained Basic Reproductive Ratio for both the areas are less than 1.This indicates that the occurrence of the disease will decrease or the disease will die out .

\section{CONCLUSIONS}

Here we have highlighted a technique for analyzing the dynamical properties of epidemiological systems through the bifurcations that they undergo at their various equilibrium points. The SIR model has been taken as a tool to estimate the chicken pox spreading in two areas of Kamrup (M) district of Assam, India. It is seen that the model helps to clearly analyze the time taken for obtaining the optimized recovery rate as well as to have a statistics of total infected people. This model will be used to optimize different parameters related to disease modeling.

\section{REFERENCES}

[1] Johnson T. 2009. Mathematical Modeling of Diseases:Susceptible-Infected-Recovered(SIR) Model.

[2] Rodrigues H. S., 2016. Application of SIR epidemiological model : new trends.

[3] Jenkins, David M. 2015. AN Examination of Mathematical Models for Infectious Disease, University of Akron: Ohio's polytechnic university, Honors Research Projects.

[4] Rahman, Ashrafur SM 2016. Study of Infectious Diseases by Mathematical Models: Predictions and Controls, Electronic Thesis and Dissertation Repository.

[5] Getz, Wayne M., and James O. Lloyd-Smith. 2005.Basic Methods for Modeling the Invasion and Spread of Contagious Diseases .DIMACS Series in Discrete Mathematics and Theoretical Computer Science.

[6] Bauch, Chris, and David E. 2003. Interepidemic Intervals in Forced and Unforced SEIR models." Dynamical Systems and Their Applications in Biology.

[7] Devi R and Dev choudhury B, 2018. SIR Model For Transmission of Chickenpox in Kamrup(Metro) District of Assam. 